\title{
La muerte en el materialismo filosófico de Gustavo Bueno
}

\section{Death in Gustavo Bueno's Philosophical Materialism}

\author{
DAVID ALVARGONZÁLEZ \\ Universidad de Oviedo (España)
}

Recibido: 19.11.2018

Aceptado: 07.10.2019

RESUMEN

Tras una breve introducción al materialismo filosófico de Gustavo Bueno y a su antropología, se expone su teoría acerca de la diferencia entre individuo humano y persona, y se distingue entre la muerte del individuo humano biológico y el fallecimiento de la persona humana. Se pasa, entonces, a presentar la diferencia entra las normas éticas, morales y políticas, con el objeto de discutir el significado de una muerte buena y un buen fallecimiento desde cada uno de esos tres sistemas axiológicos. Por último, se expone y discute, tanto desde un punto de vista ético como político, la teoría de la «eutanasia procesal» propuesta por Gustavo Bueno.

\section{PALABRAS CLAVE \\ MUERTE; FALLECIMIENTO; INDIVIDUO; PERSONA; EUTANASIA; EUTANASIA PROCESAL}

\begin{abstract}
After a brief introduction to Gustavo Bueno's philosophical materialism and to his anthropology, I will present his theory about the differences between human individual and human person, and I will put forward Bueno's distinction between the death of the biological human individual and the passing away of the human person. Later on, I will state the difference between ethical, moral and political norms, in order to discuss the meaning of a good death and a good passing away in each of those three axiological senses. Finally, I will put forward and I will discuss Gustavo Bueno's theory of «procedural euthanasia» both from an ethical and political standpoint.
\end{abstract}




\section{KEYWORDS \\ DEATH; PASSING AWAY; INDIVIDUAL; PERSON; EUTHANASIA; PROCEDURAL EUTHANASIA}

\section{EL SISTEMA DEL MATERIALISMO FILOSÓFICO}

Desde PRINCIPIOS DE LOS AÑos SESENTA del pasado siglo, hasta su muerte ocurrida en el año 2016, Gustavo Bueno desarrolló un sistema de filosofía al que él mismo se refirió con el rótulo de «materialismo filosófico». Esta etiqueta pretende subrayar el carácter materialista de su filosofía, frente al idealismo, al espiritualismo, al monismo y al formalismo, a la vez que intenta marcar distancias con el materialismo positivista de tradición marxista, tal como éste cristalizó en el materialismo histórico y dialéctico soviético. Como es sabido, Bueno contribuyó decisivamente a la defensa de la filosofía académica, al reivindicar el carácter sustantivo de la filosofía como un saber de segundo grado que supone la existencia previa de una multiplicidad de saberes de primer grado: técnicas, ciencias, tecnologías, artes, y saberes prácticos sociales, lingüísticos, y culturales, entre otros ${ }^{1}$. Bueno construyó el núcleo de su sistema filosófico en torno a una ontología y a una gnoseología o filosofía de la ciencia. Su ontología cubre tanto el ámbito de la llamada ontología general, entendida como una disciplina esencialmente crítica frente al monismo, como el de la ontología especial, donde propuso su teoría de los tres géneros de materialidad (frente a los formalismos) y su idea de ego trascendental ${ }^{2}$.

Bueno bautizó su filosofía de la ciencia con el nombre de «teoría del cierre categorial» ${ }^{3}$. Se trata de una gnoseología pluralista que parte de la multiplicidad de las ciencias, y que subraya sus componentes materiales, corpóreos, operatorios, y su carácter eminentemente sintético y constructivo. Las ciencias son los saberes de primer grado más firmes de los que disponemos, y que tenemos que tomar siempre como punto de partida para cualquier análisis filosófico, pero es necesario entender críticamente su funcionamiento para poder discernir lo que son auténticas verdades

\footnotetext{
1 Bueno, G.: Elpapel de la filosofía en el conjunto del saber. Madrid: Ciencia Nueva, 1970; Bueno G.: ¿Qué es filosofía? Oviedo: Pentalfa, 1995.

2 Bueno, G.: Ensayos materialistas, Madrid: Taurus, 1972. Bueno, G.: Materia, Oviedo: Pentalfa, 1990; Bueno, G.: El ego trascendental, Oviedo: Pentalfa, 2016.

3 Bueno, G.: Teoría del cierre categorial, 5 vols., Oviedo: Pentalfa, 1992-1993; Bueno, G.: ¿Qué es la ciencia? Oviedo: Pentalfa, 1995.
} 
científicas, es decir teoremas y principios científicos probados, de lo que son afirmaciones hechas por algunos científicos sobre cuestiones disputadas o sobre asuntos extracientíficos. En especial, Gustavo Bueno dedicó muchos esfuerzos a estudiar el estatuto gnoseológico de las llamadas «ciencias humanas» con el objeto de poder entender y discutir los límites internos de su cientificidad ${ }^{4}$.

Desde ese marco ontológico y gnoseológico materialista, Gustavo Bueno desarrolló una filosofía de la religión según la cual las fuentes de las primeras religiones en el paleolítico superior habría que buscarlas en las relaciones de los grupos humanos con otros seres reales dotados de inteligencia y voluntad con los cuales los hombres tendrían relaciones etológicas verdaderas: estas inteligencias finitas son los animales que Bueno llamó «numinosos». De acuerdo con Bueno, «el hombre hizo a Dios a imagen y semejanza de los animales» ${ }^{5}$. Una parte muy importante de sus escritos desde el año 1991 hasta su muerte han estado dedicados al desarrollo de una filosofía política materialista con extensas monografías sobre las categorías de las ciencias políticas, sobre las ideas de izquierda y derecha política, sobre España y su historia política, sobre la guerra y el terrorismo, sobre la globalización y sobre la democracia ${ }^{6}$. Gustavo Bueno ha desarrollado también una filosofía materialista de la cultura, de la televisión, del deporte, de las artes y de otros muchos asuntos y problemas del presente ${ }^{7}$. Para Bueno la actividad filosófica debe ser sistemática de modo que es

4 Bueno, G.: Estatuto gnoseológico de las ciencias humanas, 4 vóls., Madrid: Fundación Juan March, 1976.

5 Bueno, G.: El animal divino, Oviedo: Pentalfa, 1985; Bueno, G.: Cuestiones cuodlibetales sobre Dios y la religión, Madrid: Mondadori, 1989.;Bueno, G.: La fe del ateo, Madrid: Temas de Hoy, 2007.

6 Bueno, G.: Primer ensayo sobre las categorías de las ciencias políticas, Logroño: Cultural Rioja, 1991; Bueno, G.: El mito de la izquierda: las izquierdas y la derecha, Barcelona: Ediciones B, 2003; Bueno, G.: El mito de la derecha, Madrid: Temas de Hoy, 2008; Bueno, G.: España frente a Europa, Barcelona: Alba editorial 1999; Bueno, G. España no es un mito: claves para una defensa razonada, Madrid: Temas de Hoy, 2005; Bueno, G.: Zapatero y el pensamiento Alicia: un presidente en el país de las maravillas, Madrid: Temas de Hoy, 2006; Bueno, G.: La vuelta a la caverna: terrorismo guerra y globalización, Barcelona: Ediciones B, 2004; Bueno, G.: El fundamentalismo democrático. La democracia española a examen, Barcelona: Temas de Hoy, 2010; Bueno, G.: Panfleto contra la democracia realmente existente, Madrid: La Esfera de los Libros, 2004. 7 Bueno, G.: El mito de la cultura: ensayo de una teoría materialista de la cultura, Barcelona: Editorial Prensa Ibérica, 1997; Bueno, G.: Televisión, apariencia y verdad, Barcelona: Gedisa 2000; Bueno, G.: Ensayo de una definición filosófica de la idea de deporte, Oviedo: Pentalfa, 2014. 
necesario coordinar el tratamiento de los diferentes problemas filosóficos, ontológicos, gnoseológicos, éticos, morales, políticos, estéticos, de filosofía de la religión, de filosofía de la historia, etc. La sistematicidad del proceder filosófico implica, además, la consideración crítica y la discusión de las filosofías alternativas, ya que la actividad filosófica se entiende como esencialmente dialéctica.

\section{LA ANTROPOLOGÍA FILOSÓFICA DEL MATERIALISMO}

Dentro del sistema del materialismo filosófico, Gustavo Bueno también trató con mucho detenimiento los asuntos que tradicionalmente se engloban bajo el rótulo de «antropología filosófica». La antropología filosófica de Bueno tiene un momento gnoseológico que incluye la discusión del estatuto de los saberes de primer grado sobre el hombre, tal como nos son dados por las ciencias. La clasificación de las ciencias propuesta por Bueno en dos grupos, las 'ciencias no humanas ni etológicas' (física, química, biología) frente a las 'ciencias humanas y etológicas' (antropología cultural, lingüística, psicología, sociología, economía política, e historia, entre otras), es muy importante para poder analizar la muerte desde el materialismo filosófico ${ }^{8}$. Como se verá en los párrafos siguientes, la idea de individuo humano está construida fundamentalmente como una idea biológica cuya referencia es un organismo animal individual corpóreo que tiene unas determinadas características genéticas y fenotípicas. La muerte del individuo humano, en cuanto proceso biológico irreversible, no muestra diferencias significativas respecto de la muerte de otros animales emparentados filogenéticamente con el hombre. La construcción de la idea de persona, sin embargo, exige tomar en consideración los contenidos de las

8 Bueno, G.: Etnología y utopía. Respuesta a la pregunta ¿Qué es la Etnología?, $1^{\mathrm{a}}$ ed. Valencia: Azanca, 1971; $2^{a}$ ed. con un amplio «Epílogo» Madrid: Júcar, 1987; Bueno, G.: Ensayo sobre las categorías de la Economía Politica, Barcelona: La Gaya Ciencia, 1972; Bueno, G.: Estatuto gnoseológico de las ciencias humanas, 4 vóls., Madrid: Fundación Juan March, 1976; Bueno, G.: El individuo en la historia. Discurso inangural del curso 1980-81 de la Universidad de Oviedo, Oviedo: Servicio de Publicaciones de la Universidad de Oviedo, 1980; Bueno, G.: «La etología como ciencia de la cultura» en El Basilisco, 9, 1991, 3-37; Bueno, G.: «Consideraciones relativas a la estructura y a la génesis del campo de las 'ciencias psicológicas' desde la perspectiva de la teoría del cierre categorial» en III Simposium de Metodología de las Ciencias Sociales y del Comportamiento (Actas), Universidad de Santiago de Compostela, 1994, pp. 17-56. 
ciencias humanas, ya que la persona humana aparece in medias res en una sociedad de personas dotadas de lenguaje humano de palabras, y de normas y estructuras culturales e históricas: grupos de parentesco, estructuras sociales, ciudades, Estados, derecho, y tantas otras cosas. El fallecimiento de la persona tiene lugar en el contexto de su inserción en esas estructuras supraindividuales. Por esta razón, la parte gnoseológica de la antropología filosófica estará continuamente ejercitada cuando se establece la distinción entre individuo humano y persona humana, y entre muerte y fallecimiento. Desde la teoría del cierre categorial, las ciencias humanas y etológicas tienen un estatuto gnoseológico especial que afecta al núcleo de la ciencia misma, es decir, al modo de darse las verdades científicas (los teoremas y los principios). Esta circunstancia ha de tenerse en cuenta cuando se construyen ideas a partir de los conceptos y las verdades de estas ciencias ya que las verdades de las ciencias humanas y etológicas no alcanzan el grado de cientificidad propio de las ciencias no humanas ni etológicas.

Por otra parte, la antropología filosófica de Bueno tiene un momento ontológico en el que se evita hablar de la idea de hombre como una entidad existente previamente y a la que se le van añadiendo predicados: animal racional, ser religioso dotado de alma inmortal, fabricante de herramientas, ser neoténico, superhombre, por referirme aquí solamente a las cinco antropologías de predicados que expuso Max Scheler'. Desde el pluralismo ontológico y gnoseológico, la idea de «materiales antropológicos» propuesta por Bueno está pensada precisamente para no dar por supuesta la unicidad monista de un hombre del que se predican ciertas características, sean éstas las que sean. Bueno ordena la pluralidad de los materiales antropológicos por medio de un espacio de tres ejes. De acuerdo con la interpretación del espacio antropológico propuesta por David Alvargonzález, dos de esos ejes recogen las relaciones de los hombres con otros sujetos operatorios, ya sean humanos (el eje que llama «circular»), ya sean animales no humanos (en el eje «angular»); el tercer eje («radial») recoge las relaciones de los hombres con los entes inanimados, entre los que se incluyen las plantas ${ }^{10}$. Este espacio antropológico resulta significativo no

9 Scheler, M.: La idea del hombre en la historia, Buenos Aires: Ed. La Pléyade, (1924) 1974. 10 Bueno, G.: «Sobre el concepto de 'espacio antropológico'» en El Basilisco, 5, 1978, pp. 57-69; Bueno, G.: «Ensayo de una teoría antropológica de las ceremonias» en El Basilisco, 16, 1984, pp. 8-37; Bueno, G.: Nosotros y ellos. Ensayo de reconstrucción de la distinción emic/ etic de Pike, Oviedo: Pentalfa, 1990; Alvargonzález, D.: «El problema de la verdad en las reli- 
sólo por lo que afirma sino, en especial, por lo que niega.

En primer lugar, niega que para hablar filosóficamente del hombre (y de su muerte) haya que referirse a un espacio teológico, a un espacio presidido por el Dios de las religiones monoteístas teológicas y poblado de ángeles, de espíritus, de formas separadas de la materia, y de almas incorpóreas. En el materialismo filosófico de Gustavo Bueno el tratamiento filosófico de los materiales antropológicos no puede dar cabida a contenidos trascendentes, sean éstos de la naturaleza que sean. La negación del monismo, del espiritualismo y del formalismo, vistos como metafísicos, es una característica irrenunciable del materialismo filosófico como materialismo ontológico y metodológico, e implica negar la existencia de personas incorpóreas (divinas, angélicas, espirituales).

La teoría del espacio antropológico niega, además, que para tratar filosóficamente los problemas que tienen que ver con el hombre sea suficiente la perspectiva psico-etológica, es decir un espacio donde las relaciones entre individuos humanos y entre animales queden igualadas o ecualizadas, un espacio en el que los ejes circular y angular se confunden. Este espacio etológico es en el que se mueven las teorías que se fundan exclusivamente en las categorías biológicas (incluyendo la sociobiología y la genética de la conducta) pues la muerte de los hombres es, como hemos dicho antes, desde el punto de vista biológico, idéntica a la de muchos de los animales estudiados por la zoología. Este espacio etológico es también el utilizado desde las categorías de la psicología animal comparada y la etología ya que, desde el punto de vista de estas disciplinas, la conducta de los animales humanos no sería más que una variante, si acaso algo más compleja, de las conductas que apreciamos en otros animales. Esta reducción del ámbito de la antropología a la etología es la que permite a los promotores del proyecto Gran Simio hablar de personas para referirse a orangutanes, gorilas y chimpancés ${ }^{11}$.

III. INDIVIDUO Y PERSONA, MUERTE Y FALLECIMIENTO

Gustavo Bueno defendió reiteradamente la necesidad de distinguir las ideas de individuo y persona, y elaboró una teoría relacional trascenden-

giones del Paleolítico», en VV.AA. Filosofía y cuerpo: debates en torno al pensamiento de Gustavo Bueno, Murcia: Ediciones Libertarias, pp. 213-243.

11 Singer, P. y Cavallieri, P.: Elproyecto gran simio: la igualdad más allá de la humanidad, Madrid: Trotta, 1998 (original 1993). 
tal materialista de la persona humana ${ }^{12}$. Esta teoría niega rotundamente la existencia de un alma humana inmortal que sobreviva a la muerte del individuo, ya que la imposibilidad esencial de seres vivientes incorpóreos es un principio científico biológico indudable que es asumido plenamente por la ontología del materialismo filosófico, y que supone negar la posibilidad de personas divinas o angélicas incorpóreas. En el campo de la biología, el individuo humano queda definido como un organismo individual, indivisible, independiente, y dotado de una identidad genética y de una individualidad somática: desde esta perspectiva, el individuo humano es un animal más del campo de la zoología. La cuestión del momento exacto en el que comienza la vida humana individual ha sido discutida en el seno de la escuela materialista a propósito del aborto. Gustavo Bueno defendió la existencia del individuo humano desde el primer momento de la fertilización, mientras que David Alvargonzález supone que, aunque la identidad genética se constituye en ese momento, la individualidad somática sólo se alcanza cuando la gemelación deja de ser posible, es decir, en los momentos previos a la implantación ${ }^{13}$. En el momento del nacimiento, el individuo humano alcanza su plena independencia orgánica respecto de la madre; su independencia operatoria la irá consiguiendo en el proceso de crecimiento y maduración.

El recién nacido se irá convirtiendo progresivamente en una persona humana en un proceso de enculturación que supone su incorporación a la sociedad de personas que le rodea, asumiendo nuevos papeles y normas.

12 Bueno, G.: «Lectura tercera: Individuo y persona» en El sentido de la vida, seis lecturas de filosofía moral, pp. 115 -237, Oviedo: Pentalfa, 1996.

13 Bueno, G.: «La cuestión del aborto» en ¿Qué es la bioética? Oviedo: Pentalfa, 2001, pp. 87-90; Alvargonzález, D.: La clonación, la anticoncepción y el aborto en la sociedad biotecnológica, Oviedo: Pentalfa, 2009; Bueno, G.: «La ley de plazos del aborto» en El fundamentalismo democrático, Madrid: Planeta, 2010, pp. 275-321; Alvargonzález, D.: «El comienzo del individuo humano y el aborto provocado» en El Catoblepas 97, 2010, p.10. Bueno, G.: «La cuestión del aborto desde la perspectiva de la teleología orgánica» en El Catoblepas 98, 2010, p.2; Alvargonzález, D.: «Acerca del aborto y la teleología orgánica. Respuesta a Gustavo Bueno», en El Catoblepas 99, 2010, p.17; Alvargonzález, D. «Towards a nonethics-based consensual public policy on abortion», en The International Journal of Health Planning and Management, 32/1, 2015, pp. e39-e46; Alvargonzález, D.: «The constitution of the human embryo as substantial change», en Journal of Medicine and Philosophy 41/2, 2016, pp. 172-191; Alvargonzález, D.: «La idea de posibilidad en la filosofía de Gustavo Bueno», en Scientia Helmantica IV/7, 2017, pp. 112-140. 
Es un proceso largo en el que se suelen marcar ciertas etapas (infancia, uso de razón, adolescencia, juventud y mayoría de edad), y en el que la identidad personal se va construyendo en la interacción de ese individuo biológico con su entorno. Por eso la categoría de individuo humano es esencialmente biológica (genética, somática, anatómica y fisiológica), frente a la categoría de persona humana que es cultural, histórica, ética y moral.

La muerte del individuo humano tiene lugar cuando el organismo humano individual se convierte en un cadáver y empieza una fase irreversible de descomposición. Las personas, a diferencia de los individuos no nacen ni mueren. Surgen gradualmente en ese proceso de aculturación, y fallecen o desfallecen como personas, en algunas ocasiones con anterioridad a la muerte biológica del organismo. De un perro decimos que se murió mientras de una persona decimos que falleció. Esta diferencia recogida en muchos idiomas modernos entre la muerte de los animales y el fallecimiento de las personas resulta relevante. La muerte implica el paso del individuo vivo al estado de cadáver, pero no hay cadáveres de personas. Cuando las personas fallecen, se truncan las relaciones con sus familiares y amigos, y con el resto de las personas, permaneciendo sólo el recuerdo del fallecido y los resultados de sus obras.

La característica de ser una persona no se limita a unos rasgos biológicos individuales, sino que implica la inserción en un mundo cultural, histórico, moral, ético, de personas en donde se es reconocido por los demás. Ser una persona tampoco es un asunto puramente psicológico individual, una cuestión de conciencia, de voluntad, de inteligencia o de idiosincrasia de carácter: un animal salvaje puede ser inteligente y consciente, y tener apetitos y personalidad psicológica propia y, sin embargo, no es una persona. Una persona, además, puede sufrir trastornos psicofisiológicos que afecten a su conciencia (anosognosia o amnesia transitoria, prosopagnosia, etc.) y continúa siendo una persona.

La situación normal, canónica, es que exista una correspondencia biunívoca entre cada individuo humano y cada persona humana. David Alvargonzález estudió la existencia de situaciones en las que esa correspondencia biunívoca no se da: estas situaciones nos permiten entender mejor la diferencia entre las ideas de individuo y persona. La siguiente tabla muestra algunas de estas situaciones ${ }^{14}$.

14 Una versión previa de esta tabla puede verse en Alvargonzález, D.: «Alzheimer’s disea- 


\section{Tabla 1}

Falta de coordinación biunívoca entre el individuo humano y la persona humana

\begin{tabular}{|c|c|c|}
\hline Individuo & Persona & Ejemplos \\
\hline 1. Dos individuos & Una persona & Madre gestante \\
\hline 2. Dos individuos & Casi una persona & $\begin{array}{l}\text { Trastorno psicótico compartido } \\
(D S M-I V)\end{array}$ \\
\hline \multirow[t]{2}{*}{ 3. Un individuo } & \multirow[t]{2}{*}{ Dos personas } & Siameses inseparables \\
\hline & & Trastornos disociativos \\
\hline \multirow[t]{3}{*}{ 4. Un individuo } & \multirow[t]{3}{*}{ Persona $\varepsilon$} & Anencefalia \\
\hline & & Parálisis cerebral severa \\
\hline & & Muerte cerebral \\
\hline \multirow[t]{2}{*}{ 5. Un individuo } & \multirow[t]{2}{*}{ Persona ética $\varepsilon$} & Criminales convictos \\
\hline & & $\begin{array}{l}\text { Muerte vudú: eutanasia psi- } \\
\text { cosomática }\end{array}$ \\
\hline 6. Un individuo & Persona que tiende a $\varepsilon$ & Enfermedad de Alzheimer \\
\hline 7. Un individuo & $\begin{array}{l}\text { Persona formándose des- } \\
\text { de } \varepsilon\end{array}$ & Niño recién nacido \\
\hline $\begin{array}{l}\text { 8. Pérdida casi total } \\
\text { del control opera- } \\
\text { torio }\end{array}$ & Una persona & Síndrome del cautiverio \\
\hline $\begin{array}{l}\text { 9. Desaparición del } \\
\text { individuo }\end{array}$ & $\begin{array}{l}\text { Permanencia de la obra de } \\
\text { la persona }\end{array}$ & Héroes culturales \\
\hline
\end{tabular}

1. La primera situación se refiere a la madre gestante en la que reconocemos dos sujetos humanos individuales distintos, con identidad genética diferente y con individualidad somática propia, dados sus ritmos de crecimiento y de homeostasis diferentes. Sin embargo, de acuerdo con la caracterización propuesta, sólo reconocemos una persona, la madre, ya que el embrión y el feto no son personas.

2. La segunda situación recoge el caso que en DSM-IV (Diagnostic Manual of Mental Disorders) queda descrito como «trastorno psicótico compartido» y en el CIE10 (Clasificación Internacional de Enfermedades) como «trastorno delirante inducido» en el que dos individuos parecen compartir un mismo estado psicológico y personal.

3. La situación más clara en la que se puede hablar de un solo individuo orgánico y dos personas es el caso de los siameses bicípetes inseparables. Las descripciones que dan

se and euthanasia» en Journal of Aging Studies, 26, 2012, p. 378. En la versión que aquí se propone se añade el caso número uno en el que se contempla la posibilidad de la existencia de dos individuos y una sola persona. 
los psiquiatras de ciertos trastornos disociativos de la personalidad, en los que un individuo presenta varias personalidades en conflicto, podría ser otro ejemplo más discutible.

4. Este caso recoge las situaciones en las que un organismo individual humano, debido a una enfermedad grave, no puede alcanzar la condición de persona canónica, como ocurre en los casos de anencefalia y de parálisis cerebral severa. También se encuadran aquí las personas que quedan en la situación de muerte cerebral mientras su organismo continúa manteniendo las constantes vitales básicas. Se utiliza la letra griega épsilon para referirse a ese grado mínimo de constitución personal.

5. Existe un debate que no puede darse por cerrado acerca de si a los criminales convictos y confesos de crímenes horrendos se les puede retirar la condición de personas. Es muy frecuente referirse a ellos como bestias o animales. En algunas sociedades, como en el caso de la llamada muerte vudú, a estos sujetos se les aplica una especie de «eutanasia psicosomática» en la que el grupo celebra su funeral y, a partir de ese momento, actúa ignorando la existencia del sujeto, aislándolo y conduciéndolo al ostracismo hasta que muere ${ }^{15}$. En el último apartado de este artículo se discute este asunto a propósito de la eutanasia procesal.

6. La sexta situación recogida en el cuadro es el caso de un individuo canónico cuya persona desfallece progresivamente, y sus relaciones personales se deterioran como consecuencia de una enfermedad degenerativa cerebral irreversible, como por ejemplo la enfermedad de Alzheimer y otros tipos de demencia, mientras el resto de su organismo individual sigue funcionando con relativa normalidad. En las fases finales de la enfermedad de Alzheimer el paciente entra en una especie de «estado de zombi». En este caso, el fallecimiento de la persona antecede a la muerte del individuo.

7. Como ya he adelantado unas líneas más arriba, los niños recién nacidos no son todavía personas, aunque sí son individuos humanos. Se podría pensar que la persona surge desde el momento en que los padres adoptan las medidas conducentes a procrear, y toman las medidas adecuadas para la concepción y el crecimiento del individuo que luego se convertirá en persona: si fuera así, podría decirse que la persona aparece como proyecto de la sociedad de personas de la que forman parte los padres antes de que surja el individuo.

8. Un caso especialmente grave de falta de coordinación biunívoca entre individuo y persona ocurre en el llamado «síndrome del cautiverio» cuando una persona normal, como consecuencia de un accidente traumático, pierde completamente el control sobre su cuerpo de cabeza para abajo, pero sigue manteniendo su actividad cerebral y su identidad personal.

9. En el caso de los héroes culturales, una vez que estos mueren, su persona puede seguir causando efectos sobre otras personas, puede seguir «ganando batallas» a través de los proyectos que ellos promovieron o de los resultados de sus obras. Los proyectos y las obras de unas personas y otras están entrelazados: esta es la razón por la cual la muerte de las personas queridas nos afecta objetivamente, pues los cursos de esos proyectos entrelazados se truncan, y exigen a los deudos continuar sus proyectos en solitario.

Desde los presupuestos ontológicos del materialismo filosófico la vida del individuo humano termina con la muerte, de modo que los sentidos

15 Canon, W.: «Voodoo death» en American Anthropologist, 44, 1949, pp. 169-181; Lester, D.: «Voodoo death» en Omega, 59/1, 2009, pp. 1-18. 
efectivos que los sujetos van dando a sus vidas tienen que ser inmanentes a la vida humana misma. Los sentidos de la vida trascendentes serían puras ilusiones. De acuerdo con la teoría del espacio antropológico que ya he citado, los sentidos inmanentes, efectivos, de la vida de una persona humana tienen que estar dados en la religación de esa persona con otras personas, en la religación de la persona con otros contenidos no operatorios, naturales o culturales, o en la religación de la persona humana con otras inteligencias y voluntades corpóreas finitas que son los animales. Estos tres tipos de religación (circular, radial y angular) son los que contribuyen a la constitución del sujeto como persona: los sentidos de la vida de esa persona y sus proyectos vitales incluirían siempre, en proporciones variables, componentes de esos tres tipos ${ }^{16}$.

\section{LA DISTINCIÓN ENTRE ÉTICA, MORAL Y POLÍTICA}

Gustavo Bueno ha reivindicado reiteradamente la necesidad de diferenciar con claridad tres ámbitos normativos distintos que inciden sobre la praxis humana, y que él ha optado por llamar ética, moral y política. En todo caso, Bueno no trata de proponer una simple distinción terminológica acerca de los usos de ciertas palabras, sino de abrir una discusión acerca de la estructura de la realidad antropológica misma. Por ello, lo importante es argumentar la necesidad de distinguir esos tres ámbitos normativos, con independencia de que elijamos unas palabras u otras para referirnos a ellos. La distinción de estos tres ámbitos no tiene una finalidad estipulativa, prescriptiva, sino más bien pretende describir una situación de hecho. Por tanto, para criticar esta distinción será necesario evaluar su fertilidad a la hora de analizar ciertos problemas antropológicos del presente, como pueda ser el problema de la muerte de los individuos y el fallecimiento de las personas humanas que nos ocupa en este texto. Con todo, la elección de las palabras para referirse a estos tres ámbitos (ética, moral, política) trata de respetar algunos de los usos reconocidos en la discusión filosófica en el español y en otros idiomas modernos que utilizan palabras equivalentes que tienen el mismo origen griego y latino ${ }^{17}$.

16 Bueno, G.: «Religación y religión» en Bueno, G.: Cuestiones cuodlibetales sobre Dios y la religión, Madrid: Mondadori, 1989, pp. 193-228.

17 Bueno, G.: El sentido de la vida, seis lecturas de filosofía moral. Pentalfa: Oviedo, 1996; Bueno, G.: ¿Qué es la Bioética? Oviedo: Pentalfa, 2001; Bueno, G.: «En nombre de la ética» en 
La idea que subyace a esta distinción tal como la propone Gustavo Bueno es que la persona humana, en su vida diaria, se encuentra sumergida en un conjunto complejo de normas que pueden clasificarse en tres tipos, de acuerdo con los fines que persiguen: las normas éticas son las que buscan la buena marcha de la persona humana individual, las normas morales tratan de regular la buena marcha de ciertos grupos a los que las personas perteneces voluntaria o necesariamente, y las normas políticas regulan la buena marcha del Estado político. La diferencia de objetivos que persiguen cada uno de estos tipos de normas hace que, en algunas circunstancias, surjan conflictos entre ellas.

Como acabo de decir, las normas éticas son aquellas que tratan de preservar y proteger la integridad del sujeto humano, tanto tomado como organismo biológico individual como en su consideración en cuanto que persona humana. Bueno justifica la elección terminológica recordando que la palabra griega «ethos», de donde viene la española «ética», significa «carácter», y permite rescatar el aspecto distributivo individual y personal de las normas éticas. Gustavo Bueno toma de la Ética de Espinosa la virtud ética fundamental que es la fortaleza: cuando se aplica a uno mismo, la fortaleza es firmeza, cuando se aplica a otros, es generosidad. Desde esta perspectiva, la medicina, que trata de ayudar a los pacientes a reestablecer la firmeza socavada por la enfermedad, sería una profesión esencialmente ética. Las conductas antiéticas serían todas aquellas que atentan contra la integridad individual y personal: el asesinato, la tortura, la mutilación, la agresión, el rapto, la violación, el abuso, la extorsión, el chantaje, la difamación, la injuria, el insulto, el etiquetado, los tratos vejatorios, la humillación, el ultraje, la ofensa, el desprecio, la discriminación, la intimidación, la objetualización, el acoso, etc. De acuerdo con este criterio, también serían contrarias a la ética las conductas autodestructivas como el abuso de drogas, el tabaquismo, el alcoholismo, los hábitos conductuales insanos, y el suicidio. Los principios de la ética materialista prescriben el respeto de la integridad individual de toda persona humana con independencia de su edad, sexo, cultura, idioma, religión, creencias, etc. La norma ética fundamental de salvaguardar la integridad individual y personal puede conducir a conflictos como los que ocurren en la defensa propia: en el caso límite la

El Catoblepas, 16, 2003, p. 2; Bueno, G.: «Ética, Moral, Bioética, Derecho» en El Catoblepas 93, 2009, p. 2. 
protección de la vida de una persona que es atacada violentamente justificará la actuación antiética contra el atacante.

Según Bueno, las normas morales son aquellas que están diseñadas para salvaguardar la buena marcha de ciertos grupos humanos. Las personas formamos parte de muchos grupos, ya sea necesaria, ya sea voluntariamente. Ejemplos de grupos que se nos imponen son los de sexo, edad, cultura, lengua, clase social, familia, estado de salud o de enfermedad. Ejemplos de los segundos pueden ser confesiones, partidos, mafias, empresas, profesiones, instituciones, grupos de terroristas o traficantes, y tantos otros. Bueno justifica la elección terminológica en la circunstancia de que la palabra «moral» viene del latín «mores» que significa «costumbres», filogenia que permitiría utilizar esta palabra para referirse a las costumbres, a los usos, a las normas de cada grupo. Es común referirse a la moral victoriana, o burguesa, o cristiana, etc. La virtud moral principal es la lealtad y el vicio mayor la traición: la lealtad puede, en ocasiones, entrar en conflicto con las normas éticas, como cuando se trata de encubrir al colega terrorista, ladrón, abusador, corrupto o, simplemente, negligente. La norma moral que prohíbe a los Testigos de Jehová recibir una transfusión de sangre es una norma antiética ya que, dada la necesidad de recibir sangre ajena, la norma pone en riesgo la integridad del individuo. Las normas morales de grupos distintos pueden también colisionar como cuando un policía se ve en la circunstancia de tener que denunciar a un pariente.

En el mundo actual, todos los individuos humanos son ciudadanos de un Estado político y, por tanto, se ven afectados por las normas de su Estado que se consagran en la legislación. Hay un consenso bastante generalizado, y con una asentada tradición en la historia, en caracterizar como "político» todo lo que tienen que ver con los Estados. Esas normas políticas también pueden entrar en conflicto con las normas éticas y con las morales. Ejemplo de lo primero es la norma que exige al soldado matar a los enemigos o la que exige al policía impedir la entrada del emigrante ilegal. Ejemplo de lo segundo son todas las normas que el Estado establece para contener los abusos que podrían llevar a cabo determinados grupos (económicos, empresariales, religiosos, ideológicos, estamentales, etc.) y que podrían ser perjudiciales para el Estado. Por supuesto, las normas de Estados diferentes pueden colisionar entre sí como es frecuente en el ámbito de la política internacional. 
Las normas éticas, debido a que se aplican al sujeto individual tomado distributivamente, sin importar su edad, condición, sexo, etc., son normas universales. Las normas morales y políticas, sin embargo, son normas particulares de cada grupo y de cada Estado, ya que grupos diferentes tienen normas diferentes, y Estados diferentes tienen sistemas jurídicos diferentes.

\section{LA BUENA MUERTE Y LOS CONFLICTOS ENTRE ÉTICA, MORAL Y POLÍTICA}

Como ya he dicho anteriormente, el materialismo filosófico es incompatible, por razones ontológicas, con la afirmación de la existencia de vivientes incorpóreos, de inteligencias separadas (ángeles, demonios), y de todo tipo de entidades trascendentes. Así pues, la muerte del individuo humano, como ocurre con el resto de los animales, supone su desaparición, su fin consuntivo. Como ya dije, el fallecimiento de la persona humana puede anteceder a la muerte del individuo, como ocurre, en gran parte, en las enfermedades degenerativas cerebrales. Como también ha quedado dicho, la influencia de la persona que fallece no desaparece abruptamente ya que se puede dejar sentir posteriormente, en mayor o menor medida, como las ondas de un lago en el que se arroja un objeto.

Los hombres de los que se ocupa la antropología filosófica tienen lenguajes humanos de palabras lo que les permite tener una representación verbal (y luego escrita) de sus operaciones: esta circunstancia es esencial para distinguir la praxis específicamente humana, involucrada en esas representaciones verbales, y la conducta que es propia de animales no humanos. La conducta animal no humana, en ausencia de representación, se agota en su propio ejercicio. Por otra parte, como es bien sabido, el radio y la complejidad de la memoria de los individuos de nuestra especie no tienen parangón en otras especies próximas. Estas dos características distintivas (el lenguaje humano de palabras, que permite la representación verbal, y el aumento y la complejidad de la memoria) hacen posible que el hombre tenga una experiencia, una memoria y una representación de las muertes de los demás hombres que le rodean, y que van acaeciendo, y hacen que pueda ser consciente de la inevitabilidad de la muerte propia. La muerte tiene siempre tintes sombríos: el cadáver se descompone y huele mal, y todos los proyectos que incluían a la persona interfecta en su relación con los sujetos próximos quedan truncados o alterados, lo cual es un motivo 
objetivo de desdicha para los allegados supervivientes. La conciencia de la propia muerte y la evidencia de su proximidad pueden ser también un motivo de aflicción y de ansiedad. En último término, la muerte es inevitable como ya anunciaba la premisa del silogismo fúnebre: «todos los hombres son mortales».

La pregunta por las condiciones de la buena muerte, sin embargo, no tiene una respuesta única. En primer lugar, porque «muerte» puede, en unos contextos, referirse a la muerte del individuo humano y, en otros contextos, referirse al fallecimiento de la persona: por ejemplo, las condiciones en las que se da la muerte de los individuos enfermos de Alzheimer remite a un asunto muy diferente del de las condiciones en las que se da el progresivo fallecimiento de su persona. En segundo lugar, porque el adjetivo «bueno», aplicado a la muerte y al fallecimiento, tiene múltiples significados. Una muerte puede ser mala desde un punto de vista ético, y ser heroica desde un punto de vista político: por ejemplo, la muerte sangrienta de un joven guerrero en combate defendiendo a su patria. Otra muerte será mala desde el punto de vista ético y psico-fisiológico, pero buena y heroica desde el punto de vista moral: este es el caso de la muerte por ahogamiento del joven capitán que con su sacrificio logra salvar la vida de los tripulantes de su barco tras un naufragio. La muerte de Jesucristo, horrible y plagada de sufrimientos, es presentada como buena desde el punto de vista religioso pues, al parecer, ese cruento sacrificio humano habría servido para redimir al resto de los hombres de sus pecados. Si fuera así, podría entenderse como una muerte movida por el imperativo ético de la generosidad. Por el contrario, una muerte puede ser calificada como buena por el mero hecho de no llevar aparejado dolor alguno y, sin embargo, ser mala desde un punto de vista ético y moral: así sería la muerte de una madre que se suicida con analgésicos y drogas, en medio de una sensación intensa de placer, dejando huérfano a su hijo recién nacido, anteponiendo su egoísmo suicida a la generosidad para con el hijo, y destrozando a su familia ${ }^{18}$.

La «muerte digna», que sirve de motto a ciertas sociedades que pro-

18 Bueno, G. (bajo el pseudónimo de Pedro Belarmino): «La eutanasia» en El Basilisco 7, 1991, pp. 69-89. Este texto se encuentra reproducido en Bueno, G.: El sentido de la vida, seis lecturas de filosofía moral. «Lectura tercera: Individuo y persona. Apartado XI: Muerte y fallecimiento. El problema de la eutanasia» (pp. 200-237), Pentalfa: Oviedo, 1996; Bueno, G.: «La eutanasia desde una perspectiva bioética» en ¿Qué es la Bioética? Oviedo: Pentalfa, 2001, pp. 128-133; Bueno, G.: «Eutanasia» (video; 23 de agosto de 2002, 57 minutos). 
mueven la asistencia al suicidio (Dying in Dignity Society en Reino Unido, Association for the Right to Die with Dignity en Bélgica, entre otras), no puede entenderse exclusivamente como la muerte sin dolor o la muerte estética. La muerte de ese soldado, de ese capitán, la muerte de Jesucristo, sin ser analgésicas ni estéticas, son prototipos de muertes dignas. Recíprocamente, como he dicho, una muerte analgésica y estética puede ser indigna desde el punto de vista ético o moral. Por otra parte, cuando se trata de la muerte de ciertos enfermos incurables, es necesario subrayar que la circunstancia de que su muerte sea honorable depende fundamentalmente del trato que los supervivientes otorguen al moriturus, depende de que el moriturus sea tratado como una persona hasta el final, y no se le trate como a un número o a un animal. En todo caso, no depende del enfermo el ser tratado dignamente por los demás, como tampoco es culpa del enfermo el ser víctima de una enfermedad concreta.

Gustavo Bueno ha criticado también la idea de «calidad de vida» cuando se usa para justificar la inducción al suicidio. Como es sabido, en la gestión de muchas industrias es frecuente distinguir entre la cantidad de productos fabricados y su calidad. Los sociólogos y los economistas han «aplicado» esa idea a la vida misma, y hablan de «calidad de vida» de los países y de las ciudades: para ello toman nota del número de teatros, de piscinas, de bares, etc., y elaboran sus índices de calidad de vida «científicos». El siguiente paso ha consistido en aplicar esa idea de «calidad de vida» a la vida del individuo humano: algunos médicos, y otros tecnólogos de la salud, no han dudado en dar ese paso, asimilando la vida de una persona humana a un objeto producido en serie y, de este modo, objetualizando y despersonalizando esa vida humana. Esos mismos expertos a veces llegan a la conclusión de que, si una vida humana se deteriora por debajo de ciertos niveles de calidad, entonces no merece la pena seguir invirtiendo recursos médicos en su atención y su sanación. Así, se empieza a generar un clima en el que se considera que la vida de ese paciente no tiene una mínima calidad, que no merece la pena ser vivida, y se va creando a su alrededor una atmósfera de caritativa compasión, hasta que la propia «autonomía» del paciente le conduzca a solicitar el suicidio asistido ${ }^{19}$. Por ejemplo, a juicio de algunos, la vida de un tetrapléjico estaría por debajo de esos niveles mínimos de calidad de vida. Ni que decir tiene que, si las

19 Ibidem. 
personas más enfermas acceden a morir sin dilación, la calidad de vida de la población global restante, en términos estadísticos, aumentará ${ }^{20}$. Las personas con enfermedades crónicas o incurables pueden llegar a ser una carga objetiva para sus familias y cuidadores.

En muchas culturas prehispánicas de América central y meridional, se han descrito instituciones encargadas de ejecutar la eutanasia de los viejos y los enfermos. Entre otros grupos se pueden citar los guaycuru de la región del Gran Chaco en Argentina, Bolivia, Paraguay y Brasil, los indios cueva de la región de Darién en el este de Panamá, los huicholes de la Sierra Madre de Méjico, los indios bororo de la región del Mato Grosso del Brasil central, los puelches de la región de Puelmapu de los Andes, los fueguinos de Tierra del Fuego en el extremo más meridional de Sudamérica y los tucumanos del noroeste argentino. En muchas de estas culturas la persona que realiza la eutanasia es llamada «despenador», es decir, el que quita las penas o sufrimientos al enfermo administrándole la muerte. Es frecuente que esa tarea sea considerada un deber para con los familiares y amigos y que se califique de cobarde a aquel que no es capaz de afrontarla. Se apela a la familia, la amistad y el honor (valores morales) para obligar al despenador a hacer su cometido. Podemos considerar estos casos como ejemplos en los que el conflicto entre los valores éticos y morales se resuelva a favor de los valores morales, ya que la buena marcha del grupo y su viabilidad pasa por encima de la vida del individuo desvalido, enfermo o anciano.

Del mismo modo, la práctica militar extendida de matar a los enemigos gravemente heridos no sería tanto una virtud ética de generosidad o de compasión, sino una virtud moral que reconoce que, en la premura de la guerra, no hay tiempo ni recursos para cuidar de esos enfermos. En las sociedades de tradición reformada, ha llamado la atención la posición de la baronesa Mary Warnock, asesora del gobierno británico, que ha hecho un llamamiento público a las personas ancianas que sufren demencia, animándolas al suicidio, al afirmar que tienen un «deber moral de morir» para evitar sufrimientos a los familiares, y costos al sistema nacional de salud ${ }^{21}$. Esta propuesta choca con la norma general de intentar evitar el suicidio

20 Bueno, G.: «Sobre el concepto de 'calidad de vida'» en Julio Bobes, Calidad de vida y Oviedo, Oviedo: Pentalfa, 1994, pp. 9-18; El Catoblepas 169, 2016, p. 2.

21 Cfr., Warnock, M.: «A duty to die?» en Life and Work, 2008. 
de otras personas instándoles a recobrar su firmeza perdida o quebrada. Como se sabe, las sociedades que estudia la etnografía y la historia difieren en el tratamiento de la vida y la muerte humana: unas tienen institucionalizado el infanticidio preferencial femenino y otras no, unas promueven el aborto y otras lo tratan de evitar, unas tienen despenadores, mientras que en otras se intenta cuidar a las personas hasta que les llega la muerte, con independencia de su edad o estado de postración.

\section{LA EUTANASIA PROCESAL DESDE LAS PERSPECTIVAS ÉTICA Y POLÍTICA}

La filosofía de la muerte del materialismo filosófico de Gustavo Bueno incluye un análisis de la eutanasia procesal, desde las perspectivas de la ética y de la política, aplicable a autores convictos y confesos de crímenes horrendos ${ }^{22}$.

Gustavo Bueno comienza argumentando la necesidad de discutir este asunto en el presente, con independencia de la posición que finalmente se adopte. Para argumentar la oportunidad de esta discusión, parte de la evidencia de que la mal llamada «pena de muerte» fue aceptada regularmente por las legislaciones de muchos Estados durante siglos: el Código de Hammurabi, la Biblia, las Doce Tablas, el Código de Alarico, el Fuero Juzgo, las Partidas de Alfonso el Sabio, o la Constitución española de 1876, por citar sólo algunos. Constata también que la ejecución de reos de crímenes horrendos fue defendida por todos los grandes filósofos hasta el romanticismo: Platón, Aristóteles, Cicerón, Séneca, San Buenaventura, San Agustín, Santo Tomás, Francisco Suárez, Domingo de Soto, Rousseau, Kant o Hegel, entre otros.

Muchos consideran la pena de muerte como algo propio de las dictaduras o las autocracias, y la tachan de práctica antidemocrática, olvidando

22 Bueno, G.: «Diez propuestas para el próximo milenio» en Miguel Munárriz (ed.) Cincuenta propuestas para el próximo milenio, pp. 47-95. Oviedo: Fundación de Cultura Ayuntamiento Oviedo, 1995, pp. 47-95; «Propuesta 5: Implantación de la eutanasia para asesinos convictos y confesos de crímenes horrendos» (pp.77-79); Bueno, G.: «Pena de muerte: del talión a la eutanasia procesal», Conferencia impartida en los Segundos Encuentros de Filosofía en Gijón, 1997; Bueno, G.: «La eutanasia desde una perspectiva bioética» en ¿Qué es la Bioética? Oviedo: Pentalfa, 2001, pp. 128-133; Bueno, G.: «La pena de muerte como una contradicción no resuelta en la democracia» en Panfleto contra la democracia realmente existente. Madrid: La Esfera de los Libros, 2004, pp.217-224; Bueno, G.: «La cruzada contra la llamada pena de muerte» en El Catoblepas 153, 2014, p. 2. 
que está implantada en ochenta y ocho de los Estados miembros de la ONU, y que la considerada «primera democracia del mundo», los Estados Unidos de América del Norte, mantiene la pena de muerte en treinta Estados, y en los sistemas legales civiles y militares federales. Los fundamentalistas democráticos alegarán, entonces, que los Estados Unidos tienen un «déficit democrático», pero esto no deja de ser un procedimiento ad hoc de solucionar esta contradicción. Los que consideran que la discusión acerca de la conveniencia de la eutanasia procesal es una discusión arcaica, propia de salvajes, tendrán por lo menos que reconocer que su evidencia no es compartida por miles de millones de personas del presente ${ }^{23}$.

Bueno considera que las penas tienen como función principal el castigo de los actos de un sujeto que se desvían de ciertas normas, y se atiene al concepto tradicional de arrepentimiento objetivo que exige la reparación del daño infringido. El cumplimiento del castigo, y la reparación del daño, tratan de dejar claro al sujeto la necesidad de rectificar su trayectoria personal y le orientan en esa rectificación. De acuerdo con esta idea general de pena, Gustavo Bueno supone que la idea de «pena de muerte» es una idea insostenible que sólo tiene sentido desde una filosofía que defienda el animismo: sólo si se supone que el alma del ajusticiado es inmortal y sobrevive a su ejecución, se puede sostener que el alma sufre la pena de perder el cuerpo, que sería como una mutilación. Sin embargo, desde los postulados del materialismo filosófico, tan alejados del animismo, la idea de «pena de muerte» es un sinsentido ya que, si el individuo humano desaparece, no puede sufrir la pena, ni la pena puede contribuir a la re-orientación de su trayectoria personal, que queda truncada.

Bueno, en contra de la opinión hoy comúnmente admitida en muchos países europeos, considera que el mecanismo de rectificación propio de la pena tiene un límite cuando estamos tratando de autores convictos y confesos de crímenes horrendos. Supone que el crimen horrendo es de tal naturaleza que el arrepentimiento objetivo supondría el suicidio, ya que la persona no podría asumir en su trayectoria vital el propio crimen cometido. El hecho frecuente de que ciertos criminales, después de cometido el crimen horrendo, no puedan soportar lo que han hecho y se suiciden, confirma que ellos mismos consideran que sus actos no pueden quedar

23 Bueno, G.: «La cruzada contra la llamada pena de muerte» en El Catoblepas 153, 2014, p. 2. 
integrados en su trayectoria personal. Bueno sostiene que la persona que ha cometido crímenes horrendos se convierte en una "persona cero», deja de ser persona, y no puede ser reconocida por los demás como persona. Supone que este tipo de criminales han caído en un nivel tal de indignidad ética que es objetivamente irrecuperable.

Se trata, entonces, de acabar con la vida de ese individuo humano, no porque su cuerpo esté en un estadio terminal irreversible, sino porque se supone que su vida, aunque biológicamente sea una vida sana e incluso plena, desde el punto de vista ético es una vida indigna, ya que, debido a los actos antiéticos abyectos que ha cometido, ha caído a un nivel de despersonalización que se considera irrecuperable. Es una situación en la que las personas que rodean al individuo viviente lo dan por fallecido como persona, como ocurría en la muerte vudú de la que nos habló Canon, y que ya he citado más arriba. La sociedad de personas considera persona al individuo recién nacido que no tiene uso de razón, aunque, en sentido estricto no es persona en acto. Del mismo modo que la persona se constituye después del nacimiento del individuo, cabe considerar la situación en la que la persona fallece antes de la muerte del individuo, como en los casos de coma profundo irreversible a los que me he referido anteriormente. Bueno considera que algo parecido ocurre en los casos de despersonalización ética irreversible debida a la comisión de un crimen horrendo. $\mathrm{Mu}$ chos consideran ética la eutanasia del enfermo terminal fundada en la persistencia de un estado de deterioro físico grave e irrecuperable. De modo análogo, también cabría considerar ética la asistencia al suicidio en los casos de despersonalización irreversible: esta es la idea de eutanasia procesal que establecería que el criminal horrendo ha fallecido como persona (se ha degradado al nivel cero de personalidad) antes de haber muerto como individuo orgánico. La eutanasia procesual, por tanto, no eliminaría a una persona, sino que tan sólo causaría la muerte del individuo despersonalizado. Gustavo Bueno supone que, si el autor del crimen horrendo es consciente de lo que ha hecho, se suicidará. Si no es capaz de darse cuenta, habrá que intentar re-educarlo para que salga de ese estado de imbecilidad ética; pero cuando salga y sea consciente de su crimen, se suicidará al ser consciente de una carga tan insoportable. Si no llega a darse cuenta de su culpa, es la sociedad la que tiene que proceder. Si al criminal le falta la firmeza para suicidarse, entonces la sociedad le aplicara una muerte dulce 
para liberarle de esa carga insoportable: según Bueno, se trata, en ese caso, de tener piedad ante unos individuos que no son capaces de suicidarse, se trata de una especie de suicidio asistido para evitar un dolor ético y moral insufrible. Se postula que el sujeto que ha traspasado ciertos límites que afectan a su propia dignidad como persona debe asumir plenamente las consecuencias de sus actos suicidándose, de modo que, aunque desaparezca como individuo biológico, quede parcialmente redimido como persona por ese último acto de suicidio.

A mi juicio, esta justificación ética de la eutanasia procesal es muy débil, y entra en cierta contradicción con la idea de persona defendida por el propio Bueno en su antropología filosófica ${ }^{24}$. Según esa idea, el individuo humano se va conformando progresivamente como persona al interaccionar con otras personas, y al formar parte de una cultura intersubjetiva y objetiva específicamente humanas. El autor del crimen horrendo, que según Bueno ha perdido su condición de persona, sigue comportándose como persona, inmerso en la sociedad de personas, pues una parte importante de esa sociedad lo reconoce como tal (su abogado, sus familiares, sus amigos): por ejemplo, es un individuo que habla con otros y que sigue conjuntos de normas muy específicos en muchos órdenes de su vida. Por tanto, Bueno no puede querer decir que haya dejado de ser persona desde un punto de vista empírico, sino que más bien parece afirmar que es un determinado grupo el que tiene el deber moral de expulsarlo de la sociedad de personas, que es una parte de la sociedad la que dicta su inexistencia como persona. Sin embargo, ese deber queda simplemente enunciado como una prescripción moral, pero no queda fundamentado en ninguno de los principios éticos de los que propone Bueno en su ética materialista ${ }^{25}$. Además, incluso aunque se llegara a probar que el criminal ha dejado de ser persona, de ello no se deduce que haya que matarlo, pues tampoco matamos sin más a los animales que no son personas y que no actúan éticamente.

La cuestión de la eutanasia procesal también admite un análisis desde la esfera de la política. Es interesante subrayar que la abolición de la pena

24 Bueno, G.: El sentido de la vida, seis lecturas de filosofía moral. «Lectura tercera: Individuo y persona», Oviedo: Pentalfa, 1996. pp. 115-237.

25 Confróntese, por ejemplo, los principios de la bioética materialista en ¿Qué es la Bioética?, Oviedo: Pentalfa, 2001. 
de muerte se empieza a plantear en la época contemporánea, con el romanticismo y el agnosticismo. Todavía para Kant, que era un ilustrado, un criminal responsable de crímenes horrendos no podría vivir en la sociedad política. Merece la pena recordar que Kant, en la primera parte de la Metafísica de las costumbres, titulada Principios metafísicos del Derecho, en 1797, defiende la pena de muerte deduciéndola de la ley de talión.

Con la teoría acerca del origen popular del poder político, parece como si la sociedad civil, emancipada del trono y del altar, quisiera desprenderse de la carga de la ejecución capital de los penados. Muchas de las teorías del Estado de carácter contractualista contemporáneas, coetáneas a las sociedades del capitalismo industrial del consumo pletórico de bienes, tienden a concebir el Estado político como una agencia al servicio del individuo y, quizás por esa razón, evitan imponer la pena de muerte contra ninguno de sus ciudadanos. Esas teorías conciben el Estado como una asamblea de individuos atómicos, distributivamente considerados, que brotaran del territorio espontáneamente aislados, una teoría del Estado que rezuma un anarquismo de fondo. Desde esa concepción contractualista del Estado, «nadie hubiera podido transferir al Estado la potestad de quitar la vida a los mismos que lo han constituido» ${ }^{26}$. Surge entonces la teoría romántica de la pena como re-educación y reinserción que supone, por tanto, la negación de la ejecución capital. Se podría decir que, para los demócratas agnósticos de estos Estados capitalistas, como Dios no existe, entonces todo está permitido, incluso el crimen horrendo ${ }^{27}$.

La idea de eutanasia procesal se basa en el supuesto de que la vida humana individual propia no es un valor supremo, absoluto, metafísico. Este supuesto suele ser negado en las discusiones teóricas, pero es, de hecho, frecuentemente admitido cuando se elogia el comportamiento de los héroes, esas personas que ponen en riesgo su vida para salvar las de los demás o la de la comunidad. Por lo demás, es también común admitir que la vida biológica humana individual adquiere diferentes valores dependiendo de la trayectoria de la persona asociada a esa vida.

Desde el punto de vista político, la eutanasia procesal recoge la posibi-

26 Bueno, G.: «La pena de muerte como una contradicción no resuelta en la democracia» en Panfleto contra la democracia realmente existente. Madrid: La Esfera de los Libros, 2004, p. 219.

27 Bueno, G.: «Pena de muerte: del talión a la eutanasia procesal», Conferencia impartida en los Segundos Encuentros de Filosofía en Gijón, 1997. 
lidad de que sea la propia sociedad política, como sociedad de personas, la que ponga unos límites efectivos que no pueden ser traspasados, que se consideren infranqueables. Si el individuo comete ciertos crímenes horrendos, entonces está obligado a suicidarse y, si no es capaz de hacerlo, la propia sociedad le asiste en el suicidio, proporcionándole una muerte dulce. Esa eutanasia procesal no trata de castigar, pues como he dicho el concepto de pena aplicado a la pena de muerte es un sinsentido desde los presupuestos del materialismo filosófico. Tampoco trata de disuadir, ni busca la ejemplaridad, pues como es bien sabido la ejecución capital, por la publicidad que comporta, puede ser un aliciente para determinadas personas. Ni siquiera ha de ser vista como un modo de defensa del grupo frente al criminal para evitar que vuelva a delinquir, pues el grupo podría evitar esa reincidencia con la prisión perpetua. Por medio de la eutanasia procesal, la sociedad política sólo intenta marcar unos límites a las conductas de los ciudadanos, unos límites que no pueden ser traspasados, porque esa sociedad se ha dado a sí misma un código en el que no todo está permitido. El sujeto que traspase esos límites sabe que se enfrenta a su propia eliminación. A mi juicio, esta justificación de la eutanasia puede caracterizarse como política sólo en un sentido oblicuo. Es política porque queda recogida en las leyes del Estado, y porque está sometida a un proceso judicial, pero no es directamente política de acuerdo con la teoría de la eutaxia del Estado defendida por Gustavo Bueno, ya que no afecta a la buena marcha o a la viabilidad del Estado: existen muchos Estados políticos viables cuyo ordenamiento jurídico no contempla la pena de muerte. Además, el carácter no directamente político de esa eliminación del sujeto puede apreciarse en la existencia de instituciones parecidas en sociedades pre-estatales (pre-políticas), como es el caso de la muerte vudú, narrada por Canon, a la que me he referido anteriormente ${ }^{28}$.

En cualquier caso, es necesario recordar que la defensa que Gustavo Bueno hace de la eutanasia procesal se mueve fundamentalmente en un terreno filosófico teórico, y no persigue su implantación práctica, política, ya que Bueno se da cuenta de que los inconvenientes prácticos de su implantación pueden sobrepasar las eventuales ventajas. La discusión de este asunto es, más bien, un «experimento» para denunciar el animismo que actúa en el concepto tradicional de pena de muerte, y para sacar a la luz los

28 Canon, W.: «Voodoo death» en American Anthropologist, 44, 1949, pp. 169-181. 
componentes individualistas y psicologistas de las teorías éticas y políticas contractualistas de la reinserción ilimitada, asociadas al fundamentalismo democrático ${ }^{29}$.

\section{REFERENCIAS BIBLIOGRÁFICAS:}

Alvargonzález, D.: «El problema de la verdad en las religiones del Paleolítico», en Filosofía y cuerpo: debates en torno al pensamiento de Gustavo Bueno, Murcia: Ediciones Libertarias, 213-243. (http://www.nodulo.org/ec/2005/n037p12. htm)

Alvargonzález, D.: La clonación, la anticoncepción y el aborto en la sociedad biotecnológica, Oviedo: Pentalfa, 2009.

Alvargonzález, D.: «El comienzo del individuo humano y el aborto provocado» en El Catoblepas 97, 2010, p.10.

Alvargonzález, D.: «Acerca del aborto y la teleología orgánica. Respuesta a Gustavo Bueno», en El Catoblepas 99, 2010, p.17.

Alvargonzález, D.: «Alzheimer's disease and euthanasia» en Journal of Aging Studies, 26, 2012, pp. 377-385.

Alvargonzález, D.: «Alzheimer's disease and the conflict between ethics, morality and politics» en Journal of Alsheimer's Disease and Parkinsonism, S10 004, special issue Neurodegenerative Disorders, 2013.

Alvargonzález, D.: «Towards a non-ethics-based consensual public policy on abortion», en The International Journal of Health Planning and Management, 32/1, 2015, pp.e39-e46

Alvargonzález, D.: «The constitution of the human embryo as substantial change», en Journal of Medicine and Philosophy 41/2, 2016, pp. 172-191.

Alvargonzález, D.: «La idea de posibilidad en la filosofía de Gustavo Bueno», en Scientia Helmantica IV/7, 2017, pp. 112-140.

Bueno, G.: El papel de la filosofía en el conjunto del saber. Madrid: Ciencia Nueva 1970.

Bueno, G.: Etnología y utopía, $1^{\mathrm{a}}$ ed. Valencia: Azanca, 1971; $2^{\mathrm{a}}$ ed. con un amplio «Epílogo» Madrid, Júcar, 1987.

Bueno, G.: Ensayos materialistas, Madrid: Taurus, 1972.

Bueno, G.: Ensayo sobre las categorías de la Economía Política, Barcelona: La Gaya Ciencia, 1972.

29 Bueno, G.: «Pena de muerte: del talión a la eutanasia procesal», Conferencia impartida en los Segundos Encuentros de Filosofía en Gijón, 1997. 
Bueno, G.: Estatuto gnoseológico de las ciencias humanas, 4 vóls. Madrid: Fundación Juan March, 1976.

Bueno, G.: «Sobre el concepto de 'espacio antropológico'» en El Basilisco, 5, 1978, pp. 57-69.

Bueno, G.: El individuo en la historia. Discurso inangural del curso 1980-81 de la Universidad de Oviedo, Oviedo: Servicio de Publicaciones de la Universidad de Oviedo, 1980.

Bueno, G.: «Ensayo de una teoría antropológica de las ceremonias» en El Basilisco, 16, 1984, pp. 8-37.

Bueno, G.: El animal divino, Oviedo: Pentalfa, 1985.

Bueno, G.: Cuestiones cuodlibetales sobre Dios y la religión, Madrid: Mondadori, 1989.

Bueno, G.: Materia, Oviedo, Pentalfa, 1990.

Bueno, G.: Nosotros y ellos. Ensayo de reconstrucción de la distinción emic/ etic de Pike, Oviedo: Pentalfa, 1990.

Bueno, G. (bajo el pseudónimo de Pedro Belarmino): «La eutanasia» en El Basilisco 7, 1991, pp. 69-89. Este texto se encuentra reproducido en Bueno, G.: El sentido de la vida, seis lecturas de filosofía moral. «Lectura tercera: Individuo y persona. Apartado XI: Muerte y fallecimiento. El problema de la eutanasia» (pp. 200-237), Oviedo: Pentalfa, 1996.

Bueno, G.: Primer ensayo sobre las categorías de las ciencias politicas, Logroño: Cultural Rioja, 1991.

Bueno, G.: «La etología como ciencia de la cultura» en El Basilisco, 9, 1991, 3-37.

Bueno, G.: Teoría del cierre categorial, 5 vols., Oviedo: Pentalfa, 1992-1993.

Bueno, G.: «Sobre el concepto de 'calidad de vida'» en Julio Bobes, Calidad de vida y Oviedo, Pentalfa, Oviedo 1994, páginas 9-18. También publicado en El Catoblepas 169, 2016, p. 2.

Bueno, G.: «Consideraciones relativas a la estructura y a la génesis del campo de las 'ciencias psicológicas' desde la perspectiva de la teoría del cierre categorial» en III Simposium de Metodología de las Ciencias Sociales y del Comportamiento (Actas), Universidad de Santiago de Compostela 1994, pp. 17-56.

Bueno G.: ¿Qué es filosofía?, Oviedo: Pentalfa, 1995.

Bueno, G.: ¿Qué es la ciencia?, Oviedo: Pentalfa, 1995.

Bueno, G.: «Diez propuestas para el próximo milenio» en Miguel Mu- 
nárriz (ed.) Cincuenta propuestas para el próximo milenio, pp. 47-95. Oviedo: Fundación de Cultura Ayuntamiento Oviedo, 1995, pp.47-95.

«Propuesta 5: Implantación de la eutanasia para asesinos convictos y confesos de crímenes horrendos» (pp.77-79).

Bueno, G.: El mito de la cultura: ensayo de una teoría materialista de la cultura, Barcelona: Editorial Prensa Ibérica, 1997.

Bueno, G.: «Pena de muerte: del talión a la eutanasia procesal», Conferencia impartida en los Segundos Encuentros de Filosofía en Gijón, 1997.

Bueno, G.: España frente a Europa, Barcelona: Alba editorial 1999.

Bueno, G.: Televisión, apariencia y verdad, Barcelona: Gedisa 2000.

Bueno, G.: «La eutanasia desde una perspectiva bioética» en ¿Qué es la Bioética?, Oviedo: Pentalfa, 2001, pp. 128-133.

Bueno, G.: «La cuestión del aborto» en: ¿Qué es la bioética? Oviedo: Pentalfa, 2001, pp. 87-90.

Bueno, G.: «Eutanasia» (vídeo; 23 de agosto de 200257 minutos)

Bueno, G.: «En nombre de la ética» en El Catoblepas, 16, 2003 , p.2.

Bueno, G.: El mito de la irquierda: las izquierdas y la derecha, Barcelona: Ediciones B, 2003.

Bueno, G.: «La pena de muerte como una contradicción no resuelta en la democracia» en Panfleto contra la democracia realmente existente. Madrid: La Esfera de los Libros, 2004, pp.217-224.

Bueno, G.: La vuelta a la caverna: terrorismo guerra y globalización, Barcelona: Ediciones B, 2004.

Bueno, G.: España no es un mito: claves para una defensa razonada, Madrid: Temas de Hoy, 2005.

Bueno, G.: Zapatero y el pensamiento Alicia: un presidente en el país de las maravillas, Madrid: Temas de Hoy, 2006.

Bueno, G.: La fe del ateo, Madrid: Temas de Hoy, 2007.

Bueno, G.: El mito de la derecha, Madrid: Temas de Hoy, 2008.

Bueno, G.: «Ética, Moral, Bioética, Derecho» en El Catoblepas 93, 2009, p.2.

Bueno, G.: El fundamentalismo democrático. La democracia española a examen, Barcelona: Temas de Hoy, 2010.

Bueno, G.: «La ley de plazos del aborto» en El fundamentalismo democrático, Madrid: Planeta, 2010, pp.275-321.

Bueno, G.: «La cuestión del aborto desde la perspectiva de la teleología 
orgánica» en El Catoblepas 98, 2010, p.2.

Bueno, G.: Ensayo de una definición filosófica de la idea de deporte, Oviedo: Pentalfa, 2014.

Bueno, G.: «La cruzada contra la llamada pena de muerte» en El Catoblepas 153, 2014, p. 2.

Bueno, G.: El ego trascendental, Oviedo: Pentalfa, 2016.

Canon, W.: «Voodoo death» en American Anthropologist, 44, 1949, pp. 169-181.

Lester, D.: «Voodoo death» en Omega, 59/1, 2009, pp. 1-18.

Scheler, M.: La idea del hombre en la historia, Buenos Aires: Ed. La Pléyade, (1924) 1974.

Singer, P. y Cavallieri, P.: El proyecto gran simio: la igualdad más allá de la bumanidad, Madrid: Trotta, 1998 (1993).

Warnock, M.: «A duty to die?» en Life and Work, 2008.

David Alvargonzález realizó su tesis doctoral bajo la dirección de Gustavo Bueno, y ha sido también discípulo de Vidal Peña. Es profesor titular de filosofía en la Universidad de Oviedo, y es profesor tutor del Centro Asociado de Asturias de la UNED.

\section{Lineas de investigación:}

Filosofía de la ciencia, filosofía de la religión, ontología, y bioética.

\section{Publicaciones recientes:}

- «The constitution of the human embryo as substantial change», Journal of Medicine and Philosophy, vol 41, Núm. 2. (2016), pp. 172-191.

- «Knowledge and attitudes about abortion among undergraduate students», Psicothema, 29/4, 2017, pp. 520-526.

- «The structure of Bioethics as a pragmatic discipline», Metaphilosophy, vol 48, Núm. 4. (2017), pp. 467-483.

- «Sciences as systems», Perspectives on Science, Vol 27, Núm. 6. (2019), pp. 839860.

Dirección electrónica: dalvar@uniovi.es 
